\title{
$\beta$ Blockade and intermittent claudication: placebo controlled trial of atenolol and nifedipine and their combination
}

\author{
S A Solomon, L E Ramsay, W W Yeo, L Parnell, W Morris-Jones
}

\begin{abstract}
Objective-To determine the effects of the $\beta_{1}$ selective adrenoceptor blocker atenolol, the dihydropyridine calcium antagonist nifedipine, and the combination of atenolol plus nifedipine on objective and subjective measures of walking performance and foot temperature in patients with intermittent claudication.
\end{abstract}

Design-Randomised controlled double blind four way crossover trial.

Setting-Royal Hallamshire Hospital, Sheffield.

Subjects -49 patients $(40$ men) aged $39-70$ with chronic stable intermittent claudication.

Interventions-Atenolol $50 \mathrm{mg}$ twice daily; slow release nifedipine $20 \mathrm{mg}$ twice daily; atenolol $50 \mathrm{mg}$ plus slow release nifedipine $20 \mathrm{mg}$ twice daily; placebo. Each treatment was given for four weeks with no washout interval between treatments.

Main outcome measures-Claudication and walking distances on treadmill; skin temperature of feet as measured by thermistor and probe; blood pressure before and after exercise; subjective assessments of walking difficulty and foot coldness with visual analogue scales.

Results-Atenolol did not significantly alter claudication distance (mean change $-6 \% ; 95 \%$ confidence interval $1 \%$ to $-13 \%$ ), walking distance $(-2 \% ; 4 \%$ to $-8 \%)$, or foot temperature. Nifedipine did not alter claudication distance $(-4 \% ; 3 \%$ to $-11 \%)$, walking distance $(-4 \% ; 3 \%$ to $-10 \%)$, or foot temperature. Atenolol plus nifedipine did not alter claudication distance but significantly reduced walking distance $(-9 \% ;-3 \%$ to $-15 \%(\mathrm{p}<0.003))$ and skin temperature of the more affected foot $\left(-1 \cdot 1^{\circ} \mathrm{C} ; 0\right.$ to $\left.-2 \cdot 2^{\circ} \mathrm{C}(\mathrm{p}=0.05)\right)$. These effects on walking distance and foot temperature seemed unrelated to blood pressure changes.

Conclusions-There was no evidence of adverse or beneficial effects of atenolol or nifedipine, when given singly, on peripheral vascular disease. The combined treatment, however, affected walking ability and foot temperature adversely. This may have been due to $\beta$ blockade plus reduced vascular resistance, which might also explain the reported adverse effects of pindolol and labetalol on claudication.

\section{Introduction}

Patients with intermittent claudication commonly have coexistent angina or hypertension ${ }^{1-3}$ and are therefore often candidates for $\beta$ adrenoceptor blockers. Several uncontrolled observations have suggested that $\beta$ blockers may cause or worsen the symptoms of peripheral vascular disease, ${ }^{4-9}$ and intermittent claudication is widely regarded as a contraindication to $\beta$ blockers in ordinary practice. Formal studies of the effect of $\beta$ blockers on intermittent claudication are summarised in table $\mathrm{I} \cdot .^{10-15}$ Most of the studies found no adverse effect, but most were too small to allow adequate assessment ${ }^{16}$ and some were imperfectly controlled.

We report a double blind placebo controlled cross- over trial of atenolol, the dihydropyridine calcium antagonist nifedipine, and both drugs in combination in patients with intermittent claudication. We studied atenolol because it remains unclear from the findings of Roberts et al whether this $\beta$ blocker worsens intermittent claudication. ${ }^{15}$ Nifedipine, which acts as an arteriolar vasodilator, was included because little information is available on its effect in peripheral vascular disease. In the only placebo controlled study reported nifedipine did not alter exercise tolerance, as measured by pedal ergometry, or subjective walking ability. ${ }^{17} \mathrm{~A}$ major concern in that study was that six of 33 patients developed critical ischaemia after randomisation, but no information was given on the relation of deterioration to nifedipine or placebo. ${ }^{17}$ Atenolol and nifedipine have additive antihypertensive $^{18}$ and antianginal ${ }^{19}$ effects and are therefore often prescribed together. Patients with intermittent claudication are commonly treated with the two drugs simultaneously because of the frequent coexistence of hypertension, ischaemic heart disease, and peripheral vascular disease. ${ }^{1-3}$ Nifedipine might counteract coldness of the peripheries associated with $\beta$ blockade as it seems to increase skin temperature in peripheral vascular disease. ${ }^{20}$

\section{Patients and methods}

We recruited patients with stable intermittent claudication of at least six months' duration. All had leg claudication within $500 \mathrm{~m}$ of walking, absent or diminished peripheral pulses, and an ankle to brachial systolic pressure index $<0.9$ (mean 0.76 and 0.67 in right and left legs respectively) by Doppler ultrasonography at rest. Angiography was not required for entry. ${ }^{16}$ We excluded patients with rest pain, angina which limited exercise before claudication, recent myocardial infarction, insulin dependent diabetes, serum creatine concentrations $>200 \mu \mathrm{mol} / \mathrm{l}$, any contraindication to $\beta$ blockade, and coprescription of peripheral vasodilators, angiotensin converting enzyme inhibitors, or calcium antagonists. Treatment at constant dosage with diuretics, antiplatelet drugs, and other antihypertensive agents was allowed. Patients were asked to keep smoking habit, alcohol intake, diet, and exercise constant during the study.

Forty nine patients ( 40 men) aged $39-70$ met the study criteria. The mean duration of claudication was 32 months (range 6-180), and 31 patients had bilateral and 18 unilateral peripheral vascular disease. The site of disease was superficial femoral in 45 limbs and aortoiliac plus femoral in 19 patients. Twenty had angina and 10 hypertension. Twenty nine patients were current cigarette smokers and 19 former smokers. All patients gave written informed consent, and the study was approved by the hospital ethics committee.

Study design and treatments-The study was a randomised placebo controlled double blind four period crossover trial comparing atenolol $50 \mathrm{mg}$ twice daily, slow release nifedipine $20 \mathrm{mg}$ twice daily, atenolol $50 \mathrm{mg}$ plus slow release nifedipine $20 \mathrm{mg}$ twice daily, and placebo. After a four week single blind placebo run in period patients were allocated study 
numbers sequentially to provide a prerandomised treatment order, receiving each treatment for four weeks. Treatment periods were not separated by a washout interval. Trial drugs were dispensed as identical capsules to be taken one twice daily.

Exercise testing-Treadmill exercise tests were performed at entry, at two week intervals during the run in period, and at the end of each four week treatment period. After 30 minutes of acclimatisation treadmill exercise testing was performed on a Powerjog M4 treadmill (Sports Engineering, United Kingdom) at a gradient of $10 \%$. The maximum comfortable walking speed for each patient was determined at the pre-entry test and then held constant throughout. The mean walking speed was 2.9 (range $2 \cdot 0-4 \cdot 5) \mathrm{km} / \mathrm{h}$. Continuous electrocardiographic monitoring was performed during each test. Exercise tests were performed at the same time of day two to four hours after the morning dose of trial drug. The distance walked until the onset of pain was recorded as the claudication distance, and the distance walked until pain caused the patient to stop was recorded as the walking distance.

Measurements-Pre-exercise blood pressure was measured by a single observer (LP) as the mean of three readings supine (after five minutes' rest) and standing (for two minutes) using a Hawksley random zero sphygmomanometer on the right arm supported at heart level and recording phase $\mathrm{V}$ diastolic pressure. Post-exercise standing blood pressure was the mean of two readings taken two minutes after completion of exercise. The skin temperature of the plantar aspect of both big toes was measured at each visit with a thermistor and probe (models 4708 and 4700/4, Digitron Instrumentation, United Kingdom) after 30 minutes of acclimatisation. Subjective measurements of foot coldness, tiredness of legs, and difficulty in walking during the preceding treatment period were made with $10 \mathrm{~cm}$ calibrated horizontal visual analogue scales. Compliance was assessed by counting capsules returned and averaged $95 \%$ of those dispensed. There was no difference between treatments. Body weight and changes in smoking habit were recorded at each visit and showed no important change.

Statistics - The sample size was calculated to provide a power of $80 \%$ to show as significant at the $5 \%$ level a $20 \%$ difference in claudication distance between treatments. ${ }^{16}$ Analysis of variance was used to examine the variance related to patients $(\mathrm{df}=48)$, visit $(\mathrm{df}=3)$, treatment $(\mathrm{df}=3)$, and carryover $(\mathrm{df}=3)$. The residual variance was $n-58$, where $n=$ the number of observations; $\mathrm{n}$ was 196 with complete data but was usually 190. No adjustment was made in the analysis to allow for missing observations. Patients who withdrew from the study were included in the analysis up to the time of withdrawal. The significance of the carryover and treatment effects was assessed by the $F$ test, and no evidence suggested that carryover effects were present. When treatments differed in the $F$ test at $\mathrm{p}<0 \cdot 1$ pairs of treatments were compared by Student's paired $t$ test. Data from the treadmill tests were log transformed to satisfy the model assumptions, and this transformation was shown to be appropriate by residual and normal plots. The data are presented untransformed for clarity. One patient responded particularly badly to nifedipine on treadmill testing but the results had no overwhelming effect on the analysis and were retained. Standard errors of the mean were derived from the analysis of variance and were almost identical in the four treatment groups. For simplicity only those in the placebo group are shown in the tables.

\section{Results}

Withdrawals after randomisation-Three of the 49 patients withdrew after randomisation. One developed left ventricular failure during placebo treatment. Another suffered subjective worsening of claudication, lethargy, insomnia, and cold extremities while taking atenolol in the second study phase and had to discontinue treatment. Treadmill testing was performed after two weeks of treatment (before she stopped taking atenolol), and these results are included in the analysis. A third patient stopped taking combined treatment with atenolol plus nifedipine because of rest pain and cold extremities. No treadmill data were available for that treatment, and the analysis is therefore biased slightly in favour of combined treatment.

Claudication distance-The results for claudication and walking distances and subjective measurements of walking difficulty and tiredness of legs are shown in table II. During placebo treatment the mean distance walked to claudication was $66 \cdot 8$ (SEM 1.0$) \mathrm{m}$ and the total walking distance $113 \cdot 1(1 \cdot 0) \mathrm{m}$. When compared with placebo treatment atenolol, nifedipine, and the two drugs in combination shortened claudication distance slightly but not significantly. The mean reductions in claudication distance $(95 \%$ confidence interval) were $6 \%(-1 \%$ to $13 \%)$ with atenolol, $4 \%$ $(-3 \%$ to $11 \%)$ with nifedipine, and $6 \%(-2 \%$ to $13 \%)$ with atenolol plus nifedipine. The three active treatments did not differ significantly. Direct comparison of atenolol with nifedipine showed a non-significant $2 \%$ advantage for nifedipine. The effect of treatments on claudication distance was unrelated to the severity of claudication. In particular, there was no evidence of an adverse effect in those most severely afflicted as judged by claudication distance during placebo treatment. Patients in the lowest quartile had a mean claudication distance of $38 \mathrm{~m}$ (range $27-46 \mathrm{~m} ; \mathrm{n}=12$ ) during placebo treatment. The mean changes in claudication distance in this group were $+4 \%$ for atenolol, $+1 \%$ for nifedipine, and $-2 \%$ for combined treatment.

Total walking distance-For total walking distance atenolol and nifedipine caused slight but non-significant reductions from placebo values, averaging $2 \%$ $(95 \%$ confidence interval $-4 \%$ to $8 \%)$ and $4 \%(-3 \%$ to $10 \%$ ) respectively (table II). Direct comparison of atenolol with nifedipine showed a $2 \%$ advantage for atenolol, which was not significant. The combination of atenolol plus nifedipine reduced total walking

TABLE I-Formal studies of influence of $\beta$ blockers in patients with intermittent claudication

\begin{tabular}{|c|c|c|c|c|c|c|}
\hline Reference & No of patients & $\beta$ Blocker & Daily dose (mg) & Duration (weeks) & Trial & Claudication distance \\
\hline Reichert et $a l^{10}$ & 7 & Propranolol & $240-1600$ & 2 & Crossover, double blind & No change \\
\hline Lepantalo et $a l^{\prime \prime}$ & 28 & Various & Various & 4 & Parallel group, open & No change \\
\hline Bogaert and & $\int 10$ & Propranolol & 160 & 8 & Crossover, double blind & No change \\
\hline Clement $^{12}$ & $\{10$ & Metoprolol & 200 & 8 & Crossover, double blind & No change \\
\hline Hiatt et $a l^{13}$ & 19 & Propranolol & 120 & 2 & Crossover, double blind & No change \\
\hline Mlatt el al & 19 & Metoprolol & 150 & 2 & Crossover, double blind & No change \\
\hline \multirow{3}{*}{ Svendsen $e t a l^{14}$} & 14 & Metoprolol & 200 & 8 & Crossover, open & No change \\
\hline & $\left\{\begin{array}{l}11 \\
11\end{array}\right.$ & Acebutolol & 400 & 8 & Crossover, open & No change \\
\hline & 20 & Atenolol & 100 & 4 & Crossover, observer blind & No change \\
\hline \multirow[t]{2}{*}{ Roberts et als } & $\{20$ & Pindolol & 20 & 4 & Crossover, observer blind & Reduced \\
\hline & 20 & Labetalol & 400 & 4 & Crossover, observer blind & Reduced \\
\hline
\end{tabular}


TABLE II-Mean (SEM) results for objective and subjective measures of walking performance and temperature in patients with intermittent claudication

\begin{tabular}{|c|c|c|c|c|}
\hline & Placebo & Atenolol & Nifedipine & Atenolol + nifedipine \\
\hline No of patients & 48 & 49 & 46 & 47 \\
\hline Claudication distance $(\mathbf{m})$ & $66 \cdot 8(1 \cdot 0)$ & $62 \cdot 6$ & 63.9 & $62 \cdot 9$ \\
\hline Walking distance $(\mathbf{m})$ & $113 \cdot 1(1 \cdot 0)$ & 110.8 & $109 \cdot 0$ & $102 \cdot 4^{\star}$ \\
\hline \multicolumn{5}{|l|}{ Tired legs by visual } \\
\hline analogue scale $(\mathrm{mm})$ & $46 \cdot 1(2 \cdot 4)$ & $52 \cdot 8 \dagger$ & $54 \cdot 0 \ddagger$ & $50 \cdot 9$ \\
\hline \multicolumn{5}{|l|}{ Walking worse by visual } \\
\hline \\
\hline Left foot & $25 \cdot 5(0 \cdot 4)$ & $24 \cdot 5$ & $24 \cdot 7$ & $24 \cdot 6$ \\
\hline Right foot & $25 \cdot 8(0 \cdot 3)$ & $25 \cdot 0$ & $25 \cdot 0$ & $24.5 \S$ \\
\hline Worse foot & $25 \cdot 6(0 \cdot 4)$ & 24.9 & $24 \cdot 7$ & $24.5 t$ \\
\hline Better foot & $25 \cdot 8(0 \cdot 4)$ & $25 \cdot 3$ & $25 \cdot 2$ & $24.9^{\circ}$ \\
\hline
\end{tabular}

${ }^{\star} \mathrm{p}<0.003 v$ placebo; $\mathrm{p}<0.02 v$ atenolol; $\mathrm{p}=0.06 v$ nifedipine. sp<0.01 $v$ placebo.

$\neq \mathrm{p}<0.02 v$ placebo.

TABLE III-Mean (SEM) results for blood pressure and heart rate before and two minutes after treadmill exercise tests in patients with intermittent claudication

\begin{tabular}{|c|c|c|c|c|}
\hline & Placebo & Atenolol & Nifedipine & Atenolol + nifedipine \\
\hline $\begin{array}{l}\text { No of patients } \\
\text { Pre-excise supine. }\end{array}$ & 48 & 49 & 47 & 47 \\
\hline Systolic blood pressure $(\mathrm{mm} \mathrm{Hg})$ & $142 \cdot 2(1 \cdot 6)$ & $132 \cdot 3^{\star}$ & $137 \cdot 3+\ddagger$ & $132 \cdot 1^{\star}$ \\
\hline Diastolic blood pressure (mm Hg) & $84 \cdot 8(1 \cdot 1)$ & $77 \cdot 9 \star \ddagger$ & $81 \cdot 7+\oint$ & $74 \cdot 8^{\star}$ \\
\hline Heart rate (beats/min) & $82 \cdot 1(1 \cdot 1)$ & $66 \cdot 6^{\star}$ & 84.55 & $69 \cdot 6^{\star}$ \\
\hline \multicolumn{5}{|l|}{ Pre-exercise standing: } \\
\hline Systolic blood pressure $(\mathrm{mm} \mathrm{Hg})$ & $136 \cdot 6(1 \cdot 6)$ & $127 \cdot 5 \star \|$ & $126 \cdot 9 \star \ddagger$ & $121 \cdot 1^{\star}$ \\
\hline Diastolic blood pressure (mm Hg) & $86.4(0.9)$ & $79 \cdot 1 \star \mathbb{S}$ & $81 \cdot 6 \star \int^{\top}$ & $73 \cdot 8^{\star}$ \\
\hline Heart rate (beats/min) & $84 \cdot 7(1 \cdot 1)$ & $68 \cdot 6^{\star}$ & $87 \cdot 4 \varsigma^{\circ}$ & $71 \cdot 1^{\star}$ \\
\hline \multicolumn{5}{|l|}{ Post-exercise standing: } \\
\hline Systolic blood pressure $(\mathrm{mm} \mathrm{Hg})$ & $163 \cdot 1(2 \cdot 3)$ & $156 \cdot 4+\delta$ & $151 \cdot 1 \star \star \star\|\|$ & $141 \cdot 8^{\star}$ \\
\hline Diastolic blood pressure $(\mathrm{mm} \mathrm{Hg})$ & $87 \cdot 1(1 \cdot 0)$ & $82.4 \pi \S$ & $82 \cdot 2 \star S$ & $74 \cdot 4^{\star}$ \\
\hline Heart rate (beats/min) & $107 \cdot 8(1 \cdot 4)$ & $83 \cdot 1^{\star}$ & $111 \cdot 8+\oint$ & $84 \cdot 8^{\star}$ \\
\hline
\end{tabular}

${ }^{\star} \mathrm{p}<0.001 v$ placebo.

$\mathrm{tp}<0.05 v$ placebo.

$\neq \mathrm{p}<0.05 v$ atenolol + nifedipine.

$\| \mathrm{p}<0 \cdot 1 v$ atenolol + nifedipine.

Ip $<0.01 v$ placebo. distance significantly by $9 \%$ ( $3 \%$ to $15 \% ; \mathrm{p}<0.003)$. Walking distance was reduced more by the combination than by atenolol alone (by $8 \% ; 2 \%$ to $13 \%(\mathrm{p}<0.02)$ ) or nifedipine alone (by $6 \% ; 0$ to $12 \%(\mathrm{p}=0.06)$ ).

Subjective walking ability-In the subjective assessments of walking ability both atenolol and nifedipine increased tiredness of the legs as measured by visual analogue scale $(p=0.05$ and $p<0.02$ respectively; table II) with no significant difference between the treatments. Atenolol plus nifedipine when combined had no significant effect on leg tiredness despite the significant shortening of walking distance. There were no significant differences among treatments in difficulty in walking as measured by visual analogue scale.

Temperature changes - When compared with placebo, atenolol and nifedipine reduced the skin temperature of the toes slightly but not significantly (table II). Atenolol and nifedipine in combination reduced the temperature of the right foot by $1 \cdot 3^{\circ} \mathrm{C}(95 \%$ confidence interval 0.3 to $2.2^{\circ} \mathrm{C} ; \mathrm{p}<0.01$ ) and in the left foot by $0.9^{\circ} \mathrm{C}\left(-0.1\right.$ to $\left.1.9^{\circ} \mathrm{C} ; \mathrm{p}>0.05\right)$. Differences among the active treatments were not significant. When the skin temperature was analysed in the better and worse limbs, as judged by Doppler studies, combined treatment reduced the temperature of the worse limb by
$1 \cdot 1^{\circ} \mathrm{C}\left(0\right.$ to $\left.2 \cdot 2^{\circ} \mathrm{C} ; \mathrm{p}=0.05\right)$ and in the better limb by $0.9^{\circ} \mathrm{C}\left(-0.2\right.$ to $\left.1.9^{\circ} \mathrm{C} ; \mathrm{p}>0.05\right)$. The difference between worse and better limbs was not significant, and temperature changes with combined treatment did not differ significantly from those with atenolol and nifedipine given singly. Subjective coldness of the feet assessed by visual analogue scale showed no significant differences among treatments for either foot or for the better or worse foot. The objective reduction in temperature of the right foot with combined treatment was not paralleled by increased subjective coldness.

Blood pressure and heart rate - When compared with placebo, atenolol and nifedipine each reduced systolic and diastolic blood pressures when supine and standing before exercise and when standing after exercise (table III). These changes were similar with the two treatments and were generally highly significant when compared with placebo. Combined treatment with atenolol plus nifedipine decreased blood pressure further, both before and after exercise (table III). The falls in blood pressure with combined treatment were generally significantly larger than those with the drugs taken singly (table III). When compared with atenolol and nifedipine alone combined treatment reduced post-exercise systolic blood pressure by $15(95 \%$ confidence interval 8 to $21 ; \mathrm{p}<0.0001$ ) and 9 ( 3 to 16 ; $\mathrm{p}<0.01) \mathrm{mm} \mathrm{Hg}$ respectively and post-exercise diastolic pressure by 8 ( 5 to $11 ; \mathrm{p}<0.0001)$ and 8 (5 to 11 ; $\mathrm{p}<0.0001) \mathrm{mm} \mathrm{Hg}$ respectively. Heart rate was reduced highly significantly by atenolol alone and in combination with nifedipine (table III). Nifedipine alone did not significantly change supine or standing heart rate before exercise but significantly increased the post-exercise heart rate when compared with placebo (table III)

Blood pressure and walking performance-The relations between post-exercise blood pressure, objective and subjective measures of walking ability, and objective and subjective measures of foot temperature during treatment with atenolol plus nifedipine in combination are shown in table IV as correlation matrices using absolute values and changes from placebo values. There were no important relations of post-exercise blood pressure with claudication or walking distance or measures of temperature. The only significant correlation was between change in systolic pressure and subjective leg tiredness $(r=0 \cdot 40 ; p<0 \cdot 02)$, patients with smaller falls in systolic pressure having increased leg tiredness. This may have been a spurious significant finding given the number of tests performed. There was no correlation between treadmill walking distance and foot temperature, the two variables affected adversely by combined treatment. Of note was the lack of correlation between objective and subjective assessments. There were no significant relations between walking distance and subjective leg tiredness or between foot temperature and subjective coldness.

TABLE IV-Relations between post-exercise blood pressure and objective and subjective measures of walking ability during treatment with atenolol plus nifedipine in combination expressed as correlation matrices using absolute values and changes from placebo values

\begin{tabular}{|c|c|c|c|c|c|}
\hline & Claudication distance & Walking distance & Tired legs ${ }^{\star}$ & Temperature of right foot & Cold right foot ${ }^{\star}$ \\
\hline \multicolumn{6}{|c|}{ Absolute values } \\
\hline Systolic blood pressure & $0 \cdot 27$ & 0.30 & $-0 \cdot 14$ & $-0 \cdot 15$ & $0 \cdot 12$ \\
\hline Diastolic blood pressure & 0.07 & 0.08 & 0.07 & $-0 \cdot 16$ & 0.04 \\
\hline Claudication distance & & $0.86 t$ & $-0 \cdot 10$ & 0.21 & 0.02 \\
\hline Walking distance & & & -0.03 & $0 \cdot 26$ & $0 \cdot 10$ \\
\hline Tired legs ${ }^{\star}$ & & & & $0 \cdot 13$ & $0.43 \ddagger$ \\
\hline Temperature of right foot & & & & & $0 \cdot 14$ \\
\hline \multicolumn{6}{|c|}{ Changes from placebo values } \\
\hline Systolic blood pressure & 0.15 & -0.07 & $0.40 \ddagger$ & 0.03 & -0.07 \\
\hline Diastolic blood pressure & 0.20 & 0.03 & $0 \cdot 17^{+}$ & $0 \cdot 08$ & 0.09 \\
\hline Claudication distance & & 0.31 & -0.04 & 0.00 & -0.02 \\
\hline Walking distance & & & -0.08 & $0 \cdot 19$ & -0.22 \\
\hline Tired legs ${ }^{\star}$ & & & & 0.08 & $0 \cdot 23$ \\
\hline Temperature of right foot & & & & & $-0 \cdot 11$ \\
\hline
\end{tabular}


The dissociation was evident also when examining changes between treatments (table II). Significant subjective leg tiredness was observed with atenolol and nifedipine treatments with no change in walking distance, whereas the significant reduction of walking distance by combined treatment was not paralleled by increased leg tiredness.

\section{Discussion}

The $\beta_{1}$ selective blocker atenolol had no significant adverse effect on the claudication or total walking distance in these patients with peripheral vascular disease. The $95 \%$ confidence intervals precluded reductions greater than $13 \%$ for claudication distance and $8 \%$ for walking distance. There was no evidence of an adverse effect even in patients in the lowest quartile for claudication distance-that is, those with most severe peripheral vascular disease. Our findings for claudication distance were consistent with those of Roberts et al, who reported that atenolol caused no significant reduction in the distance to claudication. ${ }^{15}$ However, we were unable to confirm the significant reduction in total walking distance of about $17 \%$ observed with atenolol in their study, and the $95 \%$ confidence interval in our study was inconsistent with an effect of that magnitude.

The reason for the different outcome in the two studies, which were similar in design and dosage of atenolol, is unclear. However, Roberts et al may have overestimated any effect of atenolol on walking distance because of their smaller sample size. The only adverse effect of atenolol observed in our study was a significant increase in subjective leg tiredness measured by visual analogue scale, but this finding is not specific to patients with peripheral vascular disease..$^{21}{ }^{22} \mathrm{Leg}$ tiredness of a similar degree was observed with nifedipine. Atenolol caused a slight but non-significant reduction in peripheral skin temperature and a nonsignificant increase in subjective coldness of the feet. Our overall conclusion, however, is that atenolol had no important adverse effect on the symptoms of peripheral vascular disease. Results of the studies summarised in table I suggest that this conclusion may also hold for another $\beta_{1}$ selective blocker, metoprolol. We emphasise, however, that our study, with a sample size far larger than that in any previous study, was unable to exclude with confidence a reduction in claudication distance as large as $13 \%$ with atenolol. Other studies have generally been far too small to settle the question adequately. ${ }^{16}$

There have been suggestions that the dihydropyridine calcium antagonist nifedipine might improve $^{2023}$ or worsen ${ }^{2425}$ intermittent claudication. Its effects have been examined in only one controlled trial, which showed no significant change in intermittent claudication or subjective walking ability. ${ }^{17}$ Our study also showed no evidence for any beneficial or adverse effect of nifedipine on walking ability, and the $95 \%$ confidence intervals precluded changes in claudication or walking distance larger than a 3\% improvement or $11 \%$ worsening. The significant increase in subjective leg tiredness was unexpected. An increase in skin temperature and improvement in subjective coldness of the feet might have been anticipated as nifedipine increases skin temperature ${ }^{20}$ and commonly causes flushing and hotness of the skin. However, there was a non-significant reduction in skin temperature equivalent to that observed with atenolol. The overall conclusion for nifedipine is that it had no important negative or positive effect on the symptoms of peripheral vascular disease. Moreover, there is no support for the suggestion ${ }^{17}$ that it has an advantage over $\beta$ blockers, or at least over atenolol, in patients with peripheral vascular disease. The effects of nifedipine and atenolol on claudication and walking distances, foot temperature, and leg tiredness were virtually identical.

Combined treatment with atenolol plus nifedipine caused significantly larger falls in blood pressure before and after exercise than did the two drugs given singly. The combination caused a non-significant $6 \%$ reduction in claudication distance, and the $95 \%$ confidence interval precluded a reduction greater than $13 \%$. There was a significant reduction in walking distance averaging $9 \%$, with a $95 \%$ confidence interval of $3 \%$ to $15 \%$. The effect of combined treatment on walking distance was significantly greater than that of atenolol alone, and the difference from nifedipine alone approached significance. Atenolol plus nifedipine in combination also caused a significant reduction in skin temperature, which was slightly but not significantly more pronounced in the more affected limb. The only patient to develop critical ischaemia during the trial did so during treatment with the drugs in combination. The reductions in walking distance and skin temperature with combined treatment did not correlate with blood pressure or change in blood pressure after exercise. There was a pronounced dissociation between the findings of objective and subjective methods of assessment. Reduction in skin temperature was not paralleled by increased subjective coldness, and the significant reduction in walking distance was not associated with a noticeable increase in subjective leg tiredness. Subjective leg tiredness was less prominent with combined treatment than it was with atenolol or nifedipine given alone.

\section{CONCLUSIONS FROM AVAILABLE EVIDENCE}

What conclusions can be drawn from this study, previous clinical studies (table I), and experimental evidence concerning the effects of $\beta$ blockade on peripheral vascular disease? $\beta$ Blockers reduce maximal muscle blood flow in subjects without peripheral vascular disease. ${ }^{26}{ }^{27}$ This is observed alike with nonselective and $\beta_{1}$ selective blockers ${ }^{27}$ and is therefore unlikely to be caused by $\beta_{2}$ blockade in muscle vessels. ${ }^{27}{ }^{28}$ However, pindolol, which has substantial partial agonist activity, maintains maximum muscle blood flow, ${ }^{29}$ presumably through stimulation of $\beta_{2}$ receptors in muscle vasculature. Similar effects of $\beta$ blockade have been observed in the less affected limb of patients with peripheral vascular disease, ${ }^{30}$ including maintained maximal muscle blood flow with pindolol. ${ }^{31}$

The situation, however, seems different in the more severely affected limb of patients with peripheral vascular disease. $\beta$ Blockade has reduced maximal muscle blood flow in some studies ${ }^{30}$ but not in others. ${ }^{31}$ Differences between studies are possibly related to the method of inducing maximal flow or to the severity of vascular obstruction. ${ }^{31}$ In advanced disease the degree of arterial insufficiency itself probably becomes the main modulator of muscle blood flow ${ }^{31}$ as the reduction in flow is independent of ancillary properties of $\beta$ blockers such as $\beta_{1}$ selectivity, ${ }^{30}$ partial agonist activity, ${ }^{30}{ }^{31}$ or additional $\alpha$ blockade. ${ }^{31}$ These observations suggest that reduced maximum muscle blood flow in peripheral vascular disease is not a consequence of $\beta$ blockade locally in the ischaemic limb. This is supported by evidence that methyldopa reduces maximum muscle blood flow to the same extent as metoprolol in patients with peripheral vascular disease. ${ }^{32}$

It has been suggested, therefore, that any adverse effect of $\beta$ blockers on symptoms of peripheral vascular disease is likely due to reduction in cardiac output by $\beta$ blockade ${ }^{27}$ or to reduction of blood pressure regardless of the mechanism. ${ }^{32}$ Clinical studies (table I) and our study do not support this interpretation. Unequivocal worsening of intermittent claudication has been 
observed only with pindolol and labetalol. ${ }^{15}$ In our study only the combination of atenolol plus nifedipine had a significant adverse effect on walking distance, and this effect was significantly different from that of atenolol alone. These three treatments-pindolol, labetalol, and atenolol plus nifedipine-have in common their combination of $\beta$ blockade with a reduction in peripheral vascular resistance. The reduced vascular resistance is caused respectively by $\beta_{2}$ agonist activity, ${ }^{33} \alpha$ blockade, and non-specific arteriolar vasodilatation. These treatments cause less reduction in cardiac output than does $\beta$ blockade with no additional vasodilatation..$^{193}$ The view that reduced cardiac output is the important factor in worsening walking performance is therefore difficult to sustain.

Blood pressure reduction is also unlikely to be the critical influence. In this study atenolol plus nifedipine combined had a significant added hypotensive effect, as expected.$^{18}$ However, there was no relation between blood pressure or blood pressure change and the deterioration of walking performance with combined treatment. In the study of Roberts et al blood pressure reductions were similar with pindolol, labetalol, atenolol, and captopril, yet only pindolol and labetalol reduced claudication distance significantly. ${ }^{15}$ Reduction in blood pressure does not therefore seem to be the proximate cause of symptomatic deterioration in patients with peripheral vascular disease.

We propose that adding vasodilatation to $\beta$ blockade, whether through partial agonist activity, $\alpha$ blockade, or non-specific vasodilatation, may be the key factor in symptomatic worsening of peripheral vascular disease. Reduction in vascular resistance in unaffected or less affected vascular beds may lead to a local "steal" phenomenon $^{34}$ and reduce perfusion of limbs with obstructive arterial disease without affecting the blood pressure recorded at the brachial artery. In the case of pindolol the steal may be to unaffected muscle, mediated by $\beta_{2}$ receptor stimulation, ${ }^{31}$ and in the case of labetalol mainly to the skin through $\alpha$ blockade. ${ }^{31}$ In this study a significant reduction in skin temperature with atenolol plus nifedipine, particularly in the more affected leg, lends indirect support to this hypothesis. The corollary of the hypothesis is that the increase in vascular resistance observed with $\beta$ blockers which have no additional partial agonist activity, $\alpha$ blockade, or vasodilator action ${ }^{27}$ may actually be necessary to maintain local blood flow and prevent symptomatic deterioration in patients with peripheral vascular disease.

What are the implications of these observations for ordinary medical practice? We conclude that the $\beta_{1}$ selective blocker atenolol can generally be used safely in patients with intermittent claudication, and this may also be true for other $\beta_{1}$ selective blockers such as metoprolol. There is insufficient evidence to draw any firm conclusion on the safety or otherwise of propranolol and other non-selective $\beta$ blockers without partial agonist activity. Nifedipine seems to offer no advantage over atenolol in patients with peripheral vascular disease. The combination of atenolol plus nifedipine worsens walking distance and reduces skin temperature significantly in patients with intermittent claudication. $\beta$ Blockers which also reduce vascular resistance, whether by partial agonist activity, additonal $\alpha$ blocking activity, or non-specific vasodila- tation, should probably be avoided in patients with peripheral vascular disease until further evidence on their safety becomes available.

We are grateful to ICI PLC, United Kingdom, for financial support and help with the statistical analyses.

I Kannel WB, Skinner JJ, Schwartz MJ, Shurtleff D. Intermittent claudication: incidence in the Framingham study. Circulation 1970;41:875-83.

2 Hughson WG, Mann JI, Garrod J. Intermittent claudication: prevalence and risk factors. $B M \mathcal{F} 1978 ; \mathrm{i}: 1379-81$.

3 Ramsay LE. Intermittent claudication in hypertensive men. $f R$ Coll Physicians Lond 1979;13:100-2

4 Frohlich ED, Tarazi RC, Dustan HP. Peripheral arterial insufficiency. A complication of beta-adrenergic blocking therapy. $\mathcal{J} A M A$ 1969;208:2471-2. Rodger JC, Sheldon CD, Lerski RA, Livingstone WR. Intermittent claudication complicating beta-blockade. $B M \mathcal{F} 1976 ; \mathrm{i}: 1125$.

6 Vale JA, Jeffreys DB. Peripheral gangrene complicating beta blockade. Lancet 1978;i:1216.

7 Gokal R, Dorman TL, Ledingham JGG. Peripheral skin necrosis complicating beta blockade. $B M \mathcal{Y}$ 1979;i:721.

8 Fogoros RN. Exacerbation of intermittent claudication by propranolol. $N$ Engl f Med 1980;302:1089.

9 Ingram DM, House AK, Thompson GH, Stacey MC, Castleden WM, Lovegrove FT. Beta-adrenergic blockade and peripheral vascular disease. Med F A ust 1982;i:509-11.

10 Reichert N, Shibolet S, Adar R, Gafni J. Controlled trial of propranolol in intermittent claudication. Clin Pharmacol Ther 1975;17:612-5.

11 Lepantalo M, von Knorring J, Lindfors O, Scheinin DM. The effect of withdrawal of beta-adrenergic blockade on intermittent claudication. Angiology 1983;34:401-11.

12 Bogaert MG, Clement DL. Lack of influence of propranolol and metoprolol on walking distance in patients with chronic intermittent claudication Eur Hearl f 1983;4:203-

13 Hiatt WR, Stoll S, Nies AS. Effect of $\beta$ adrenergic blockers on the peripheral circulation in patients with peripheral vascular disease. Circulation 1985;72 1226-31

14 Svendsen TL, Jelnes R, Tonnesen KH. The effects of acebutolol and metoprolol on walking distances and distal blood pressure in hypertensive patients with intermittent claudication. Acta Med Scand 1986;219:161-5.

15 Roberts DH, Tsao Y, McLoughlin GA, Breckenridge A. Placebo-controlled comparison of captopril, atenolol, labetalol, and pindolol in hypertension complicated by intermittent claudication. Lancet 1987; ;i:650-3.

16 Cameron HA, Waller PC, Ramsay LE. Drug treatment of intermittent laudication: a critical analysis of the methods and findings of published clinical trials, 1965-1985. Br f Clin Pharmacol 1988;26:569-76.

17 Lewis P, Psaila JB, Davies WT, Morgan RH, Woodcock JP. Nifedipine in patients with peripheral vascular disease. Eur f Vasc Surg 1989;3:159-64.

18 Aoki K, Kondo S, Mochizuki A, et al. Antihypertensive effect of cardiovascular Ca-antagonist in hypertensive patients in the absence and presence of betaCa-antagonist in hypertensive patients in the abse

19 Findlay IN, Dargie HJ. The effects of nifedipine, atenolol and that combination on left ventricular function. Postgrad Med F 1983;59 (suppl 2):70-3.

20 Yamada T, Hiramatsu K, Nagata H, Zeni M. The efficacy of nifedipine (Adalat) towards Raynaud's phenomenon and intermittent claudication. fpn f Exp Med 1981;58:239-43.

21 Lewis RV, Jackson PR, Ramsay LE. Quantification of side-effects of $\beta$ adrenoceptor blockers using visual analogue scales. Br $\mathcal{F}$ Clin Pharmorn 1984; 18:325-30.

22 Lewis RV, Jackson PR, Ramsay LE. Side-effects of adrenoceptor blockers assessed by visual analogue scales. Br f Clin Pharmacol 1985;19:255-7.

23 Fisher MM, Snart AG. Nifedipine in intermittent claudication of peripheral arterio-sclerosis. Clin Res 1984;32:828A.

24 Lorentsen E, Landmark $K$. The acute effects of nifedipine on calf and forefoot blood flow in patients with peripheral arterial insufficiency. Angiology 1983;34:46-52.

25 DiPerri T, Forconi S, Guerrini M, Laghi Pasini F, Pecchi S, Cappelli R. Action of flunarizine and nifedipine on leg blood flow in patients with peripheral obstructive arterial disease. Int Angiol 1984:3:61-73.

26 McSorley PD, Warren DJ. Effects of propranolol and metoprolol on the peripheral circulation. BMF 1978;ii: 1598-600.

27 Lepantalo $M$, Totterman KJ. Effect of long-term beta-adrenergic-blockade on calf blood flow in hypertensive patients. Clin Physiol 1983;3:35-42.

28 Kendall MJ. Are selective beta-adrenoceptor blocking drugs an advantage? fR Coll Physicians Lond 1981;15:33-40.

29 Atterhog JH, Duner H, Pernow B. Experience with pindolol, a beta-recepto blocker, in the treatment of hypertension. Am F Med 1976;60:872-6.

30 Smith RS, Warren DJ. Effect of $\beta$-blocking drugs on peripheral blood flow in intermittent claudication. 7 Cardiovasc Pharmacol 1982;4:2-4.

31 Lepantalo M. Chronic effects of labetalol, pindolol, and propranolol on cal blood flow on intermittent claudication. Clin Pharmacol Ther 1985;37:7-12.

32 Lepantalo $\mathrm{M}$. Chronic effects of metoprolol and methyldopa on calf blood flow in intermittent claudication. Br $\mathcal{F}$ Clin Pharmacol 1984;18:90-3.

33 Man in't Veld AJ, Schalekamp MADH. Effects of 10 different $\beta$-adrenoceptor antagonists on hemodynamics, plasma renin activity, and plasma norepinephrine in hypertension: the key ref phrine in hypertension: the key role of vascular resistance changes in relation
to partial agonist activity. C Cardiovasc Pharmacol 1983;5:S30-45.

34 Gillespie JA. The case against vasodilator drugs in occlusive vascular disease of the legs. Lancet 1959;ii:995-7.

(Accepted 23 fuly 1991 